\title{
Design and Realization of AC Charging Spot for Electric Vehicles
}

\author{
Shuguang $\mathrm{Liu}^{1}$, Gang Cheng ${ }^{2}$ and Huawei $\mathrm{Xie}^{2}$ \\ ${ }^{1}$ School of Mechatronic Eng., Huangshan University, Huangshan 245041, China \\ ${ }^{2}$ School of Electronic and Information Eng., Xi' an Polytechnic University, Xi' an 710048, China
}

\begin{abstract}
Electric vehicle as a new kind of transportation vehicle has broad application prospects in our country. Charging facilities which provide energy with electric vehicles are important infrastructure in vehicle industry development. The equivalent model and working principle of $\mathrm{AC}$ charging spots are studied in this paper. A control solution based on the micro-controller STM32F107VCT6 is designed for the requirements. The design of hardware and software in the control system and the electrical part of charging spot are introduced in detail. To meet the requirements of the charging progress, the system provides a variety of functions, such as human-computer interaction, charging control, electric energy measurement, IC card payment, bill printing, running condition monitoring, charging protection and charging information storage and uploading.
\end{abstract}

Keywords-electric vehicle; AC charging spot; vehicle-mounted charger

\section{INTRODUCTION}

With the deepening of the global energy crisis, oil resources running out, as well as hazards exacerbate air pollution, global warming, Governments and automakers generally recognized energy saving and emission reduction is the direction of future automotive technology development, development of electric vehicles would be the best way to solve these two problems [1] China attaches great importance to the development of electric vehicles, the State has issued a series of policies to support and standardize the development of electric vehicles. But to make electric cars large popularity in China still has a long way to go, need to address a lot of problems. In a recent publication of the energy-saving and new energy vehicles in the draft plan that will be electric as the main strategic orientations. Experts pointed out that three bottlenecks of pure electric vehicle development: one is the lack of standards; second, the policy is not perfect; third, infrastructure planning and building in an orderly fashion. This AC charging electric vehicles as part of the charging infrastructure is of great significance for promoting the popularization of electric vehicles.

\section{Ac Charging Electric Vehicle Introduced}

AC charging, also known as AC-powered devices are fixed to the floor or wall, installed in public buildings and residential parking lot or in the charging station, transmission way for electric vehicles with the charger provides human-computer interaction interface and $\mathrm{AC}$ charging port, and have the special device for measuring and protection functions of [2]. AC charging use of large-screen LCD color touch-screen interface, you can choose to set power, time, amount, automatic (until full) four modes charging, condition monitoring and fault monitoring, charge time measurement, records and historical data storage functions. At present, the commonly used electric vehicle charging slow charging and quick and fast for three [2]:

1) Slow charge mode:

Slow filling in small AC current to recharge, charging time is typically a slow charge mode $6 \sim 10 \mathrm{~h}$ to charge every night! Night can be used when charging low electricity prices, to reduce the cost of charging, but emergency or driving long distances to meet users needs. Slow filling with single phase $220 \mathrm{~V} / 16 \mathrm{~A}$ on $\mathrm{AC}$ power, and through the car charger to charge the electric cars, car charger socket GB of three can be used, the basic interface standard is not an issue. Electric slow filling through the post.

2) The fast charge mode:

Fast charge, also known as emergency charging large DC currents within the $20 \mathrm{~min}$ to $1 \mathrm{~h}$, to charge the electric cars for short time service, the fast charge mode mileage is insufficient power supply problems can be solved, but have an impact on battery life, due to the larger, to technology, security requirements are also higher. Fast charge is characterized by high voltage and large current, charge time is short (about 1h). At present, this method of charging the charging socket pin assignments, voltage and current values, control protocols are not national standards or international standards, has been put to use Chargers and electric vehicle battery socket is defined by the manufacturer. The fast charge mode is mainly for in the charging station.

3) Quick:

Quick change through additional direct replace the car battery power, for power with a similar fuel vehicle refueling time, about need 5 10min, quick change most convenient, but you need to standardize electric car and car batteries, and fast changing needs operations professionals in the process. In this way the advantages of electric vehicle battery without charging, replace the battery for a short time, but require the complete reunification of the shape, size and other parameters of the battery at the same time, also requires structural design to meet the replacement battery for electric vehicle convenience and rapidity.

\section{AC CHARGING SYSTEM}

\section{A. Equivalent Model}

Model Structure of AC charger as shown in Figure I (a) as shown. Three-phase bridge type controllable rectifier circuit 
for three-phase alternating current of rectifier, filter for high frequency DC-DC power conversion circuit to provide DC input, power conversion circuit of outputs after the output filter, recharge the battery.

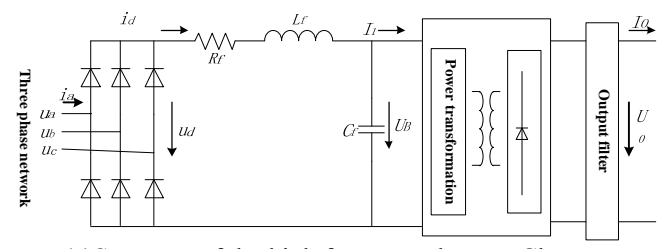

(a)Structure of the high frequency battery Charger

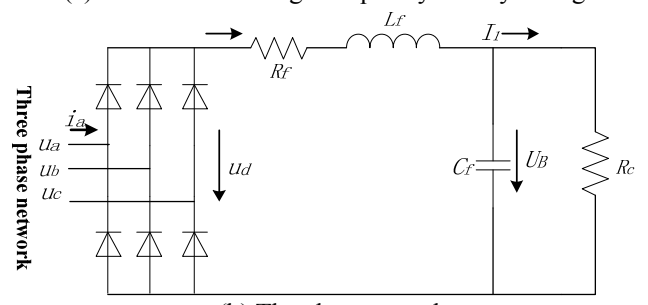

(b) The charger mode

FIGURE I. AC CHARGER MODEL

Figure I (a) equivalent power converter parts. Relative to the power frequency cycles, battery charging process takes a long time, in one to several power cycles, can think the charger output current and output voltage is constant DC, Figure I (b) $\mathrm{U}_{0}, \mathrm{I}_{0}$ is constant. Therefore, high frequency power conversion link works in constant power state when the input voltage $U_{B}$ rises, the input current $I_{1}$ must reduce, the input impedance is negative impedance characteristics. In the low frequency range, a nonlinear resistor RC can be used to simulate the equivalent input resistance of high frequency power conversion link, RC can be approximated as:

$$
R_{c}=\frac{U_{B}}{I_{1}}=\frac{U_{B}^{2}}{P_{1}}=\frac{\eta U_{B}^{2}}{P_{0}}=\frac{\eta U_{B}^{2}}{U_{0} I_{0}}
$$

Where $\eta$ is the efficiency of the charging machine.

\section{B. Working Principle}

According to GB/t 20234.2-2011 the connection device for electric vehicle conductive charging: Exchange of relevant provisions in charging interface requirements, control pilot circuit is used as the way of charging connection status and judgment device rated current parameters. Typical control pilot circuit as shown in Figure II [3].

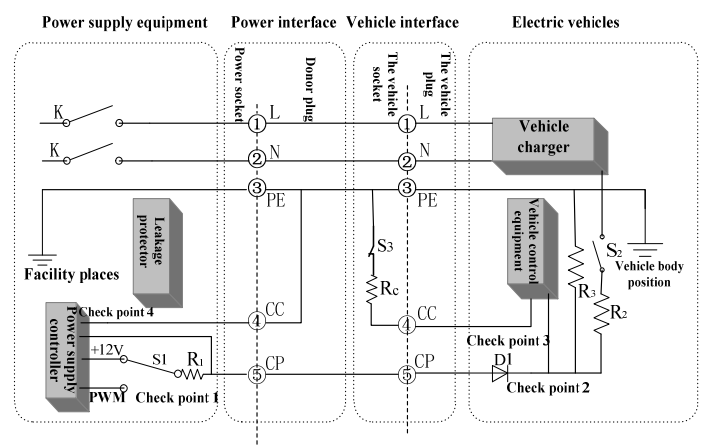

FIGURE II. TYPICAL CONTROL PILOT CIRCUIT DIAGRAM
Power supply Plug and socket connections, power supply control device by detection of point 4 as shown in Figure II voltage values determine the power supply plug from the power supply socket is fully connected. By measuring electric vehicle control device detection points at 3 , resistance values with the PE judge vehicle plug from the vehicle socket is fully connected. After the completion of the plug and socket connection state detection, power supply units charged operators to start setting, the switch S1 switch to PWM connection status from the $+12 \mathrm{~V}$ State of the connection, the power supply controls a PWM signal. Voltage of power supply control device by measuring point 1 value determines whether the charging connection is fully connected. Vehicle control test correct closure S2, power supply control device by detection of point 1 of the voltage value again determines whether the vehicle is ready to meet the requirements by closing $\mathrm{k} A C$ power supply turn-on.

\section{Composition of the Electric System}

Electrical system consists of a LCD touch screen, printer, RS485 interface meter, earth leakage protection circuit breaker, contactor, card reader and LED lights and other basic components. LCD touch screen provides a friendly human-machine interface and the quick and easy way to meet customer requirements for electric vehicle charging in different ways, you can display the current charge status, charge battery and charging fees. When the voltage exceeds the overvoltage protection of the collection value or below the under-voltage protection value, charging stops charging. Earth leakage protection circuit breaker charging, it guarantees that the leakage occurred during emergency situations, such as to stop charging. When an accident requires emergency stop charging, charging can be interrupted by emergency stop button. Systems electrical connection diagram as shown in Figure III

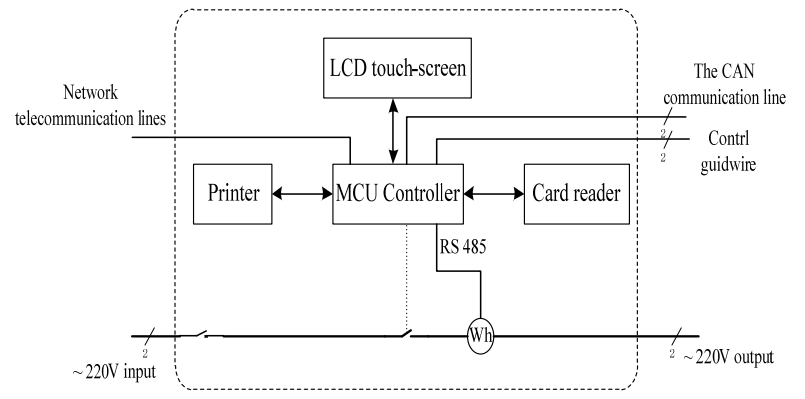

FIGURE III. ELECTRICAL CONNECTION AC CHARGING DIAGRAM

\section{Control Module Circuit Design}

\section{A. Main Controller}

Main controller select stmicroelectronics STM32F107VCT6 micro controller. Type STM32F107VC interconnected series of high-performance ARM architecture (M3 32-bit RISC core, working frequency is $72 \mathrm{MHZ}$. The device contains two 12 ADC, four general 16-bit timer and a PWM timer, also contains the standard and the advanced communication interface: as many as two I2C, three SPI, 2 I2S, five USART, 1 and 2 USB CAN, the chip provides Ethernet interface at the same time, greatly convenient for circuit design. 


\section{B. Serial Interface Circuit}

Four serial interface control module were used respectively with LCD touch screen, thermal printer, card reader, and RS485 communication interface of watt-hour meter. LCD touch screen and thermal printers for RS232 level after level conversion and MCU communication. LCD touch screen and MCU communication protocol adopts the MODBUS RTU communication protocols, MCU as a host, LCD touch screen as the machine. Thermal printer according to the printer module provides protocol to communicate. Card reader for TTL level, can be directly connected to the MCU, the card reader module provides protocol to communicate. Charge measurement of watt-hour meter adopts multifunction single-phase table, meter choose [4] 2.0 grade of watt-hour meter, electric current specifications for 5 A (40). Meters provide RS485 interface, communication protocol by DL/T 645-645 and MCU communication. By reading watt-hour meter electricity value as electricity measurement value of charging pile, by reading meter current and voltage values to determine whether any over-current and over-voltage in the process of charging, and processing. Watt-hour meter interface circuit as shown in Figure IV.

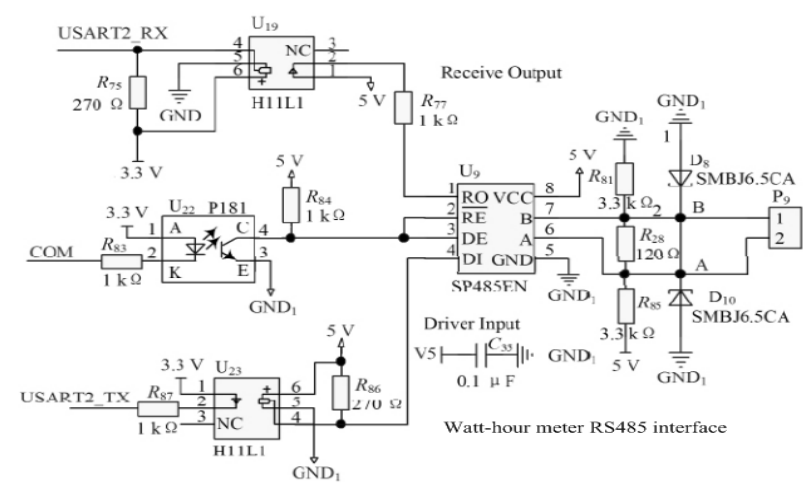

FIGURE IV. RS485 INTERFACE CIRCUIT

\section{A CAN Bus Niterface Circuit}

According to the electric cars, car charger and ac charging pile communication agreement draft in the relevant specification, the draft recommended car charger and the exchange between charging pile using CAN bus communication system, so the design of CAN bus interface. Data link layer provides the reliable data transmission between the physical connection, this system car charger and ac charging pile data frame format is in line with CAN bus between $2.0 \mathrm{~B}$ version of the regulation, use CAN extend frame 29 identifier. Specific corresponding definition of each bit allocation and transmission protocol, and other functions in accordance with the provisions of the SAE J1939-21 [5].

\section{Charging Voltage Measurement Circuit}

Voltage measurement need to first by measuring transformer converts voltage and current can be measured small signal. A survey of $220 \mathrm{v}$ voltage signal, for example, using the transformer change than $2 \mathrm{~mA} / 5 \mathrm{~mA}$, adopts the circuit shown in Figure V. At $220 \mathrm{v}$ transformer output of 5 $\mathrm{mA}$, ignoring the effect of shunt resistance, the $27 \Omega \mathrm{R}_{2}$ rather then a sampling resistance. [6] Due to the sampling signals for alternating current (AC), has A positive and negative, and A/D converter input range of $0 \sim 3.6 \mathrm{~V}$, so can't directly to sampling the voltage input to the $\mathrm{A} / \mathrm{D}$ converter. In the op-amp input access is a positive reference voltage, then choose the appropriate magnification, make the output to $\mathrm{A} / \mathrm{D}$ converter input range can be very good solve the problem. After using quasi synchronous sampling, data using rectangular since the convolution window to calculate the effective value [7].

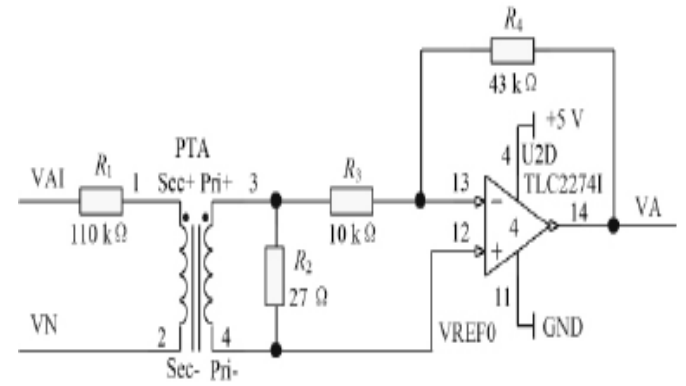

FIGURE V. CHARGING VOLTAGE MEASUREMENT CIRCUIT

\section{E. Direct Circuit Controller}

Control guidance before completing the charging circuit and electric car charging pile connection confirmation, charging power supply power and connection device identification of a current-carrying capacity and charging process of monitoring task. MCU through check points to judge in different voltage value, the circuit principle diagram is shown in Figure VI.

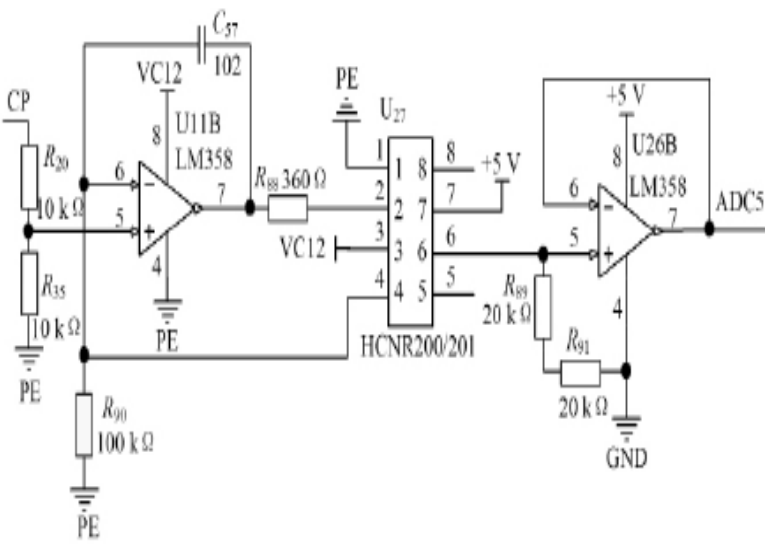

FIGURE VI. CONTROLS THE STEERING CIRCUIT

\section{F. The Software Design}

Charging pile through touch screen interactive control, runtime if read card for credit card operation, raise the interrupt determine the type of card related operations. Charging mode can provide a wide range of choice set to charge for the amount of time, energy, and can also be set directly with so far. Overall program flow chart shown in Figure VII. 


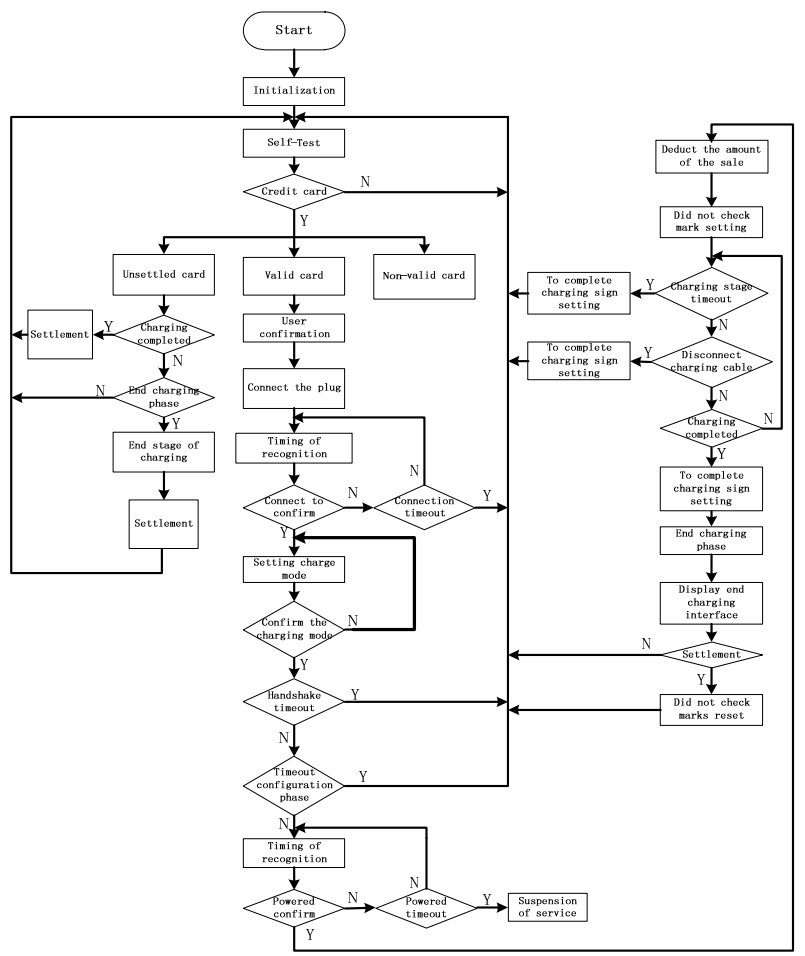

FIGURE VII. PROGRAM FLOW CHAT

\section{ELECTRICAL DESIGN}

Ac electrical part of the main charging pile complete charging control and protection function in the process of charging. With leakage protection, short circuit protection, over-current, over-voltage and under-voltage protection protection function. In addition to the short circuit and earth leakage protection, other protective functions through the charge controller control contactor, in order to realize the recovery. Short circuit and earth leakage protection with leakage protection use miniature circuit breaker. In addition, the electrical system also has lightning protection module, lightning protection module nominal discharge current not less than $20 \mathrm{kA}$, protect the voltage level is less than or equal to $1.5 \mathrm{KV}$. When the single-phase power supply lightning protection module connection mode selection of $\mathrm{P}+\mathrm{N}$ connection mode. Charging pile with abrupt stop button, so that in an emergency can forced termination of charging.

\section{CONCLUSION}

Electric vehicles is one of the future development prospect in our country traffic tools, electric vehicle charging pile provide energy supply for electric cars running, one of the important facilities necessary to develop electric vehicles. This paper analyzes the communication control module of hardware design and software design of charging pile, describes the part of the electrical charging pile design. STM32F107VCT6 as the core, the control module to realize the human-computer interaction, charging control, electric energy metering, IC card pay, paper printing, running state monitoring, charging protection and information storage and upload and so on the many kinds of perfect function. This module can satisfy the requirement of the electric car generally slow charge, as part of the charging infrastructure to promote the popularity of electric cars, has the vital significance.

\section{REFERENCE}

[1] WANG Taolumang,ZHOU Xiaobing, ZHANG Wei. The electric car charging infrastructure development both at home and abroad research [J]. Central China electric power, 2010, 23 (5) : 16-20, 31.

[2] ZHANG Qian,HAN Weijian, YU Jihui, etc. Electric vehicle charging station simulation model and its influence to power grid harmonic [J]. Journal of electrical engineering technology, 2012, 27 (2) : 159-164.

[3] General Administration of Quality Supervision,Inspection and Quarantine of P.R.C. GB/T 20234.2 2011 charging electric conduction with connection device: AC charging interface [S]. Beijing: China standard press, 2011.

[4] China southern power grid. Q/CSG11516.8-2010 Electric vehicle charging stations and charging pile acceptance specification [S]. Guangzhou:China southern power grid company, 2010.

[5] General Administration of Quality Supervision,Inspection and Quarantine of P.R.C. Electric cars, car charger and ac charging pile communication protocol between the draft [S]. Beijing: China standard press.

[6] Tai Hsien-chung.The Quasisynchronous sampling and its application in the measurement of nonsinusoidal power. [J]. Chinese Journal of scientific instrument, 1984, 5 (4) : 98-100.

[7] LIU Shuguang, LI Pengfei, HUANG Xinbo. The Quasi-synchronous Sampling and Its Algorithms on Relay Protection[C]. 2010 Asia-Pacific Power and Energy Engineering Conference, 2010, Mar., 28-31. 